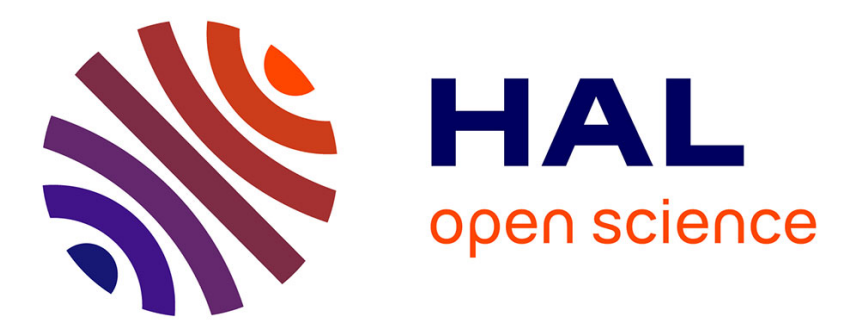

\title{
Effective permeability of anisotropic porous materials containing periodically or randomly distributed cracks
}

Viet Thanh To, Quy-Dong To, Guy Bonnet, Ba Anh Le, Thoi Trung Nguyen

\section{To cite this version:}

Viet Thanh To, Quy-Dong To, Guy Bonnet, Ba Anh Le, Thoi Trung Nguyen. Effective permeability of anisotropic porous materials containing periodically or randomly distributed cracks. European Journal of Mechanics - A/Solids, 2020, 80, pp.103902. 10.1016/j.euromechsol.2019.103902 . hal-02453271

\section{HAL Id: hal-02453271 \\ https://hal.science/hal-02453271}

Submitted on 23 Jan 2020

HAL is a multi-disciplinary open access archive for the deposit and dissemination of scientific research documents, whether they are published or not. The documents may come from teaching and research institutions in France or abroad, or from public or private research centers.
L'archive ouverte pluridisciplinaire $\mathbf{H A L}$, est destinée au dépôt et à la diffusion de documents scientifiques de niveau recherche, publiés ou non, émanant des établissements d'enseignement et de recherche français ou étrangers, des laboratoires publics ou privés. 


\title{
Effective permeability of anisotropic porous materials containing periodically or randomly distributed cracks
}

\author{
Viet-Thanh $\mathrm{To}^{\mathrm{a}}$, Quy-Dong To ${ }^{\mathrm{b}, *}$, Guy Bonnet ${ }^{\mathrm{b}}, \mathrm{Ba}^{\mathrm{A}}$ Anh Le $\mathrm{Le}^{\mathrm{c}}$ \\ ${ }^{a}$ Le Quy Don Technical University, 236 Hoang Quoc Viet, Bac Tu Liem, Ha Noi \\ ${ }^{b}$ Université Paris-Est, Laboratoire Modélisation et Simulation Multi Echelle, MSME \\ UMR 8208 CNRS, 5 Boulevard Descartes, 77454 Marne-la-Vallée Cedex 2, France \\ ${ }^{c}$ University of Communication and Transport, 3 Cau Giay, Dong Da, Ha Noi
}

\begin{abstract}
This paper aims at predicting effective transport properties of fractured porous media based on the information of cracks distribution within the material. The porous materials are assumed to contain aligned insulating and superconductive cracks, arranged in parallel layers. Two types of cracks distributions are considered: periodic and random distributions. In the former periodic case, the estimates are analytically derived from the approximation of polarization integral equations, and compare well with a numerical solution. In the latter random case, the estimates show explicit connections to planar structure factors of hard disks, a statistical quantity of phases distribution in Fourier space. Different random ensembles of hard disks are also examined to study how they affect the effective permeability of the material. Keywords: Permeability, Porous media, Cracks, Structure factor, Polarization equations.
\end{abstract}

\section{Introduction}

It is well known that artificial and natural materials like clay, rock, shale, polymer, foam, etc.. contain pores and cracks over a large range of length

\footnotetext{
${ }^{*}$ Corresponding author. Email: quy-dong.to@u-pem.fr
} 
scales. In many industrial applications, e.g hydraulic fracturing, the porous media are intentionally fractured to enhance the fluid transport. It is of prime importance to predict the permeability of these materials from the information obtained from the microstructure. In the ideal case, the tomography imaging of the Volume Representative Element (VRE) and an associated numerical model can be used to simulate flows through the internal pore and crack network to predict the transport properties. Such a task is very challenging since it requires a full knowledge of the microstructure and a huge computation cost devoted to simulation. Since the fractured porous media are usually randomly heterogeneous materials, it is relevant to use a statistical description to represent the phases, in this case the pore and crack network. Our main objective is to determine the effective transport properties of porous media containing cracks and to link the effective properties with statistical descriptors of the porous network.

Regarding the literature on the computation of effective properties of porous media with cracks, most of the works are based on the solutions of Eshelby problems (Eshelby, 1957) and the associated Hill tensors for ellipsoid voids, the cracks being obtained in the limit case of one vanishing radius of the ellipsoid(see e.g Torquato, 2001; Milton, 2002; Nemat-Nasser and Hori, 2013; Mura, 1987, and the references therein). Homogenization schemes based on Eshelby's solutions such as dilute scheme (Snow, 1969; Shafiro and Kachanov, 2000), Mori-Tanaka, self consistent (Benveniste, 1987; Fokker, 2001; Dormieux and Kondo, 2004; Barthélémy, 2009; Pozdniakov and Tsang, 2004), or differential schemes (Hashin, 1988; Zimmerman, 1996) are used to estimate the effective properties (elasticity and/or conductivity) of the materials. However, the interaction between heterogeneities is obviously simplified by using a basic solution related to isolated heterogeneities. To check the validity of such schemes, numerical models have been used, like Finite Elements (Sævik et al., 2013) or a boundary integral equation approach 
(Vu et al., 2018; Pouya and Vu, 2012) or mulipole expansion (Kushch, 1997; Kushch and Sangani, 2000). More recently, numerical schemes based on the Fourier Transform (FT) have been used as an alternative powerful numerical method (Michel et al., 1999) to provide effective properties. While the FT based methods have proved to perform efficiently in many situations, the extension of the methods to media containing cracks is still challenging. The present paper dealing with cracks is based on such a solution in the FT domain.

Concerning statistical aspects, numerous works were performed since Kröner's pioneering work (Kröner, 1977) using correlation functions of heterogeneous phases (see Torquato, 2001; Milton, 2002, and the references therein). The statistical information on the heterogeneous material can be used to derive estimates or bounds of the transport properties. However, to the best of our knowledge, the cases of porous materials with anisotropic distribution of cracks subject to the present research, have not been well studied.

To this aim, our work is based on the ability of the description of the flow in FT domain to introduce naturally statistical descriptors in Fourier space, like structure factors (Hansen and McDonald, 2006), as shown in some of our recent works related to the case of composites (Nguyen et al., 2016; To et al., 2013). This approach is of prime interest because structure factors can be obtained by several ways, either numerically or experimentally. Specifically, the structure factor has been studied in the context of physics of interacting particles (Hansen and McDonald, 2006; Percus and Yevick, 1958; Wertheim, 1963; Ornstein and Zernike, 1914) and can be obtained numerically from the Monte Carlo or Molecular Dynamics methods. While those latter methods are among the popular inverse techniques to generate random media with high porosity for computation, for example, when compared with the classical Random Sequential Addition method (RSA), their use to provide estimates 
of effective properties based on the structure factor were not considered before (Nguyen et al., 2016; To et al., 2013). Applying the same approach to cracks is not trivial due to various issues, including the vanishing volume fraction, the extreme contrast, the resolution and the singularity. Therefore, the present work aims at predicting the effective behaviour of porous materials containing cracks by resolving the above issues.

The porous materials considered in the present paper are therefore composed of families of micropores and unconnected cracks which can be treated separately. While the permeability of porous matrix can be well described by Darcy's law with a finite hydraulic conductivity, the transport behavior across the crack is either superconductive or insulating depending on the nature of the filling material. Furthermore, the geometry of the cracks, their distribution and interactions can have a significant influence on the overall permeability of the cracked porous material.

The contribution of this work focuses on the statistical aspect of transport properties of porous media. Specifically, we study how to estimate the effective permeability from the distribution of identical cracks. Starting from a periodic model (see e.g Nemat-Nasser et al., 1993; Nguyen et al., 2016; To et al., 2013; Nguyen and To, 2018), an integral equation in terms of polarization inside the ellipsoid is first established for the general case where the porous matrix is anisotropic. Then, the effective permeability is estimated, based on a direct approximation of the average polarization in the integral equation. For random distributions of cracks, we show that the estimates are directly related to the structure factor of hard discs (instead of hard spheres for spherical inclusions), a statistical quantity that describes the local distribution of cracks in Fourier space. 


\section{Problem formulation and theoretical estimates}

\subsection{Transport equations for heterogeneous anisotropic porous materials}

We study a heterogeneous and saturated porous material where the permeability or hydraulic conductivity $\boldsymbol{K}(\boldsymbol{x})$ (resistance $\boldsymbol{R}(\boldsymbol{x})$ ) is a function of coordinate $\boldsymbol{x}$. The local Darcy law can be written as

$$
\boldsymbol{j}(\boldsymbol{x})=\boldsymbol{K}(\boldsymbol{x}) \cdot \boldsymbol{e}(\boldsymbol{x}), \quad \boldsymbol{e}(\boldsymbol{x})=-\nabla \theta(\boldsymbol{x})
$$

where $\boldsymbol{j}, \theta$ and $\boldsymbol{e}$ are respectively fluid velocity, pressure and (minus) pressure gradient. To find the effective hydraulic conductivity $\boldsymbol{K}^{e}$, the general method is to apply suitable boundary conditions on a Representative Element Volume (REV) $V$ and to solve the related boundary value problem, leading to the local variables $\boldsymbol{j}, \boldsymbol{e}$. The effective conductivity $\boldsymbol{K}^{e}$ is obtained from the volume averages of $\boldsymbol{j}$ and $\boldsymbol{e}$ via the linear relation

$$
\boldsymbol{J}=\boldsymbol{K}^{e} . \boldsymbol{E}, \quad \boldsymbol{J}=\langle\boldsymbol{j}\rangle, \quad \boldsymbol{E}=\langle\boldsymbol{e}\rangle
$$

The notation \langle\rangle$_{V}$ represents the volume average over $V$. In practice, we can study rectangular box volumes $V=a_{1} \times a_{2} \times a_{3}$ with Periodic Boundary Conditions (PBC). In this case, $\boldsymbol{j}$ and $\boldsymbol{e}$ are $V$-periodic functions and can be obtained by means of Fourier Transform techniques. For example, the Fourier Transform $\phi(\boldsymbol{\xi})$ of any periodic function $\phi(\boldsymbol{x})$ is defined as

$$
\phi(\boldsymbol{x})=\sum_{\boldsymbol{\xi}} \phi(\boldsymbol{\xi}) e^{-i \boldsymbol{\xi} \cdot \boldsymbol{x}}, \quad \phi(\boldsymbol{\xi})=\frac{1}{V} \int_{V} \phi(\boldsymbol{x}) e^{-i \boldsymbol{\xi} \cdot \boldsymbol{x}} d \boldsymbol{x}
$$

where $\boldsymbol{\xi}\left(\xi_{1}, \xi_{2}, \xi_{3}\right)$ is the wave vector

$$
\xi_{i}=2 \pi n_{i} / a_{i}, \quad n_{i}=0, \pm 1, \pm 2, . ., \pm \infty, \quad i=1,2,3
$$

A classical method (Milton, 2002) is to characterize the heterogeneity effect using the polarization $\boldsymbol{\tau}(\boldsymbol{x})$ in an homogeneous material of constant conduc- 
tivity $\boldsymbol{K}^{0}$ (resistance $\boldsymbol{R}^{0}$ ) which is chosen arbitrarily. The polarization is defined by its relation with the local flux as:

$$
\boldsymbol{j}(\boldsymbol{x})=\boldsymbol{K}^{0} \cdot \boldsymbol{e}(\boldsymbol{x})+\boldsymbol{\tau}(\boldsymbol{x}),
$$

It is possible to show that $\boldsymbol{j}$ and $\boldsymbol{e}$ are connected to $\boldsymbol{\tau}$ via the Fourier space relation (Monchiet and Bonnet, 2013)

$$
\begin{aligned}
& \boldsymbol{K}^{0} \cdot \boldsymbol{e}(\boldsymbol{\xi})=-\boldsymbol{P}(\boldsymbol{\xi}) \cdot \boldsymbol{\tau}(\boldsymbol{\xi}) \quad \forall \boldsymbol{\xi} \neq \mathbf{0}, \quad \boldsymbol{e}(\boldsymbol{\xi}=\mathbf{0})=\boldsymbol{E} \\
& \boldsymbol{j}(\boldsymbol{\xi})=\boldsymbol{Q}(\boldsymbol{\xi}) \cdot \boldsymbol{\tau}(\boldsymbol{\xi}) \quad \forall \boldsymbol{\xi} \neq \mathbf{0}, \quad \boldsymbol{j}(\boldsymbol{\xi}=\mathbf{0})=\boldsymbol{J}
\end{aligned}
$$

where $\boldsymbol{P}(\boldsymbol{\xi})$ and $\boldsymbol{Q}(\boldsymbol{\xi})$ are operators defined as

$$
\begin{aligned}
& \boldsymbol{P}(\boldsymbol{\xi})=\frac{\boldsymbol{K}^{0} \cdot(\boldsymbol{\xi} \otimes \boldsymbol{\xi})}{\boldsymbol{\xi} \cdot \boldsymbol{K}^{0} \cdot \boldsymbol{\xi}} \quad \forall \boldsymbol{\xi} \neq \mathbf{0}, \quad \boldsymbol{P}(\boldsymbol{\xi}=\mathbf{0})=0 \\
& \boldsymbol{Q}(\boldsymbol{\xi})=\boldsymbol{I}-\frac{\boldsymbol{K}^{0} \cdot(\boldsymbol{\xi} \otimes \boldsymbol{\xi})}{\boldsymbol{\xi} \cdot \boldsymbol{K}^{0} \cdot \boldsymbol{\xi}} \quad \forall \boldsymbol{\xi} \neq \mathbf{0}, \quad \boldsymbol{Q}(\boldsymbol{\xi}=\mathbf{0})=0
\end{aligned}
$$

and $\boldsymbol{I}$ is the second order identity tensor. Making use of the relation $\boldsymbol{\tau}=$ $\delta \boldsymbol{K} \boldsymbol{e}=-\boldsymbol{K}^{0} \delta \boldsymbol{R} \boldsymbol{j}$ where $\delta \boldsymbol{K}=\boldsymbol{K}(\boldsymbol{x})-\boldsymbol{K}^{0}$ and $\delta \boldsymbol{R}=\boldsymbol{R}(\boldsymbol{x})-\boldsymbol{R}^{0}$, the above relations can be used to formulate the equations in physical space

$$
\boldsymbol{K}^{0} . \boldsymbol{e}=\boldsymbol{K}^{0} . \boldsymbol{E}-\boldsymbol{P} * \delta \boldsymbol{K} . \boldsymbol{e}, \quad \boldsymbol{j}=\boldsymbol{J}-\boldsymbol{Q} * \boldsymbol{K}^{0} . \delta \boldsymbol{R} . \boldsymbol{j}
$$

in which $*$ denotes the convolution operator in the physical space. These two basic equations are used in classical FFT based numerical homogenization methods (Michel et al., 1999). These equations can be transformed into equations on $\tau$

$$
\boldsymbol{\tau}=\delta \boldsymbol{K} . \boldsymbol{E}-\delta \boldsymbol{K} . \boldsymbol{R}^{0} . \boldsymbol{P} * \boldsymbol{\tau}, \quad \boldsymbol{\tau}=-\boldsymbol{K}^{0} . \delta \boldsymbol{R} . \boldsymbol{J}-\boldsymbol{K}^{0} . \delta \boldsymbol{R} . \boldsymbol{Q} * \boldsymbol{\tau}
$$


Linearly combining the two integral equations with tensor $\left(\boldsymbol{K} \cdot \boldsymbol{R}^{0}+\boldsymbol{I}\right)^{-1}$ and $\left(\boldsymbol{K} \cdot \boldsymbol{R}^{0}+\boldsymbol{I}\right)^{-1} \cdot \boldsymbol{K} \cdot \boldsymbol{R}^{0}$ yields

$$
\boldsymbol{\tau}=\boldsymbol{A} \cdot(\boldsymbol{T}+\boldsymbol{H} * \boldsymbol{\tau})
$$

with

$$
\boldsymbol{A}=\left(\boldsymbol{K} . \boldsymbol{R}^{0}+\boldsymbol{I}\right)^{-1}\left(\boldsymbol{K} . \boldsymbol{R}^{0}-\boldsymbol{I}\right), \quad \boldsymbol{H}=\boldsymbol{Q}-\boldsymbol{P}, \quad \boldsymbol{T}=\boldsymbol{K}^{0} \cdot \boldsymbol{E}+\boldsymbol{I}(11)
$$

The interest of using (10) instead of (9) is that it is more convenient to study both limit cases: superconductor $\boldsymbol{K} \rightarrow \infty$ or insulator $\boldsymbol{K} \rightarrow 0$. Unlike $\delta \boldsymbol{K} . \boldsymbol{R}^{0}$ and $\boldsymbol{K}^{0} . \delta \boldsymbol{R}$, tensor $\boldsymbol{A}$ is always bounded in these two limits (Nguyen and To, 2018).

\subsection{Estimates of the effective conductivity}

We consider two phase materials composed of a matrix of conductivity $\boldsymbol{K}^{0}$, which characterizes the reference material, and heterogeneities of conductivity $\boldsymbol{K}^{1}$. The polarization $\boldsymbol{\tau}$ vanishes in the matrix and the resulting equation becomes

$$
\boldsymbol{\tau}=\chi \boldsymbol{A} \cdot(\boldsymbol{T}+\boldsymbol{H} * \boldsymbol{\tau})
$$

Here $\chi(\boldsymbol{x})$ is the characteristic function, equal to 1 in the superconductor (insulator) areas $\Omega$ and vanishing elsewhere (matrix). From now on, we shall use the notation $\boldsymbol{A}=\left(\boldsymbol{K}^{1} \cdot \boldsymbol{R}^{0}+\boldsymbol{I}\right)^{-1}\left(\boldsymbol{K}^{1} \cdot \boldsymbol{R}^{0}-\boldsymbol{I}\right)$ instead of $\boldsymbol{A}=\chi\left(\boldsymbol{K}^{1} \cdot \boldsymbol{R}^{0}+\right.$ $\boldsymbol{I})^{-1}\left(\boldsymbol{K}^{1} \cdot \boldsymbol{R}^{0}-\boldsymbol{I}\right)$ according to relation (11). It is clear that $\boldsymbol{K}^{1} \rightarrow \infty$ corresponds to superconductor and $\boldsymbol{K}^{1} \rightarrow 0$ to insulator heterogeneity. As a consequence, one obtains:

$$
\begin{aligned}
& \text { superconductor, } \boldsymbol{A}=\boldsymbol{I}, \quad \boldsymbol{\tau}=\chi(\boldsymbol{T}+\boldsymbol{H} * \boldsymbol{\tau}) \\
& \text { insulator } \boldsymbol{A}=-\boldsymbol{I}, \quad \boldsymbol{\tau}=-\chi(\boldsymbol{T}+\boldsymbol{H} * \boldsymbol{\tau})
\end{aligned}
$$


Obtaining the value of the effective permeability needs the solution of (13). However, a good approximation (Nemat-Nasser and Hori, 2013) can be obtained as follows: averaging both sides over the heterogeneity volume $\Omega$ and considering $\boldsymbol{\tau}$ uniform over the inclusions, i.e. $\boldsymbol{\tau}=\chi\langle\boldsymbol{\tau}\rangle_{\Omega}$ when evaluating the last term of the right hand side, we obtain

$$
\langle\boldsymbol{\tau}\rangle_{\Omega}=\boldsymbol{A} \cdot\left[\boldsymbol{T}+\boldsymbol{C}\langle\boldsymbol{\tau}\rangle_{\Omega}\right]
$$

The explicit expression of $\boldsymbol{C}$ is the following

$$
\boldsymbol{C}=f^{-1} \sum_{\boldsymbol{\xi} \neq 0} \chi(-\boldsymbol{\xi}) \boldsymbol{H}(\boldsymbol{\xi}) \chi(\boldsymbol{\xi})
$$

where $f=\Omega / V$ is the volume fraction. Because $\boldsymbol{H}(\boldsymbol{\xi}=\mathbf{0})=\mathbf{0}$, the sums $\sum_{\boldsymbol{\xi} \neq 0}$ and $\sum_{\boldsymbol{\xi}}$ involving $\boldsymbol{H}(\boldsymbol{\xi})$ are identical. Due to the choice of the reference tensor, the polarization vanishes in the matrix, thus

$$
\langle\boldsymbol{\tau}\rangle_{\Omega}=f^{-1}\langle\boldsymbol{\tau}\rangle_{V}=f^{-1}\left(\boldsymbol{J}-\boldsymbol{K}^{0} . \boldsymbol{E}\right)
$$

Combining this last result with $\boldsymbol{J}=\boldsymbol{K}^{e} . \boldsymbol{E}$ and $\boldsymbol{T}=\boldsymbol{J}+\boldsymbol{K}^{0} . \boldsymbol{E}$, we can evaluate the effective tensor $\boldsymbol{K}^{e}$ from the relation

$$
(\boldsymbol{I}-\boldsymbol{A} \cdot \boldsymbol{C}) \cdot\left(\boldsymbol{K}^{e}-\boldsymbol{K}^{0}\right) . \boldsymbol{E}=f \boldsymbol{A} \cdot\left(\boldsymbol{K}^{e}+\boldsymbol{K}^{0}\right) \cdot \boldsymbol{E}
$$

and obtain

$$
\begin{aligned}
& \boldsymbol{K}^{e}=[\boldsymbol{I}-\boldsymbol{A} \cdot \boldsymbol{C}-f \boldsymbol{A}]^{-1} \cdot[\boldsymbol{I}-\boldsymbol{A} \cdot \boldsymbol{C}+f \boldsymbol{A}] \cdot \boldsymbol{K}^{0} \\
& =\boldsymbol{K}^{0}+2 f[\boldsymbol{I}-\boldsymbol{A} \cdot \boldsymbol{C}-f \boldsymbol{A}]^{-1} \cdot \boldsymbol{A} \cdot \boldsymbol{K}^{0}
\end{aligned}
$$

We note that the calculation of tensor $\boldsymbol{C}$ related to heterogeneities with finite dimensions can be obtained numerically without difficulty. However, a special treatment must be used to study the problem in the crack limit due 
to the vanishing form factor $\chi \rightarrow 0$ and volume fraction $f \rightarrow 0$. This issue will be addressed in the following. Some ingredients of the techniques can be found in (Nemat-Nasser et al., 1993).

\subsection{Ellipsoidal inclusions and cracks centered in the periodic cell}

We assume that the principal axes of the anisotropic material and of an ellipsoidal heterogeneity centered at the origin, are directed along $x_{1}, x_{2}$ and $x_{3}$. The form factor $\chi(\boldsymbol{\xi})$ of the ellipsoids with semi axes $b_{1}, b_{2}, b_{3}$ and volume $\Omega=\frac{4 \pi}{3} b_{1} b_{2} b_{3}$ is given by the expression

$$
\begin{aligned}
& \chi(\boldsymbol{\xi})=f \varphi(\eta), \quad \varphi(\eta)=\frac{3(\sin \eta-\eta \cos \eta)}{\eta^{3}}, \\
& \eta=\sqrt{\left(\xi_{1} b_{1}\right)^{2}+\left(\xi_{2} b_{2}\right)^{2}+\left(\xi_{3} b_{3}\right)^{2}},
\end{aligned}
$$

Near the crack limit, i.e $b_{3} \rightarrow 0$, the form factor can be expressed as a
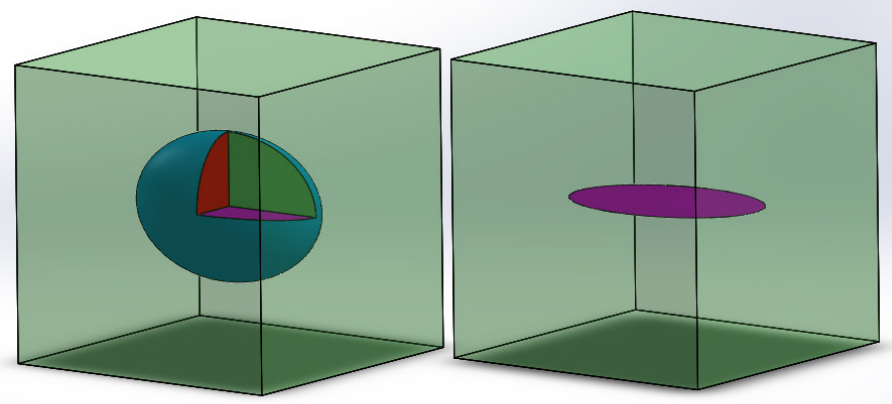

Figure 1: Ellipsoids and penny shape crack in unit cell.

function of wavevector by: $\boldsymbol{\xi}^{*}\left(\xi_{1}, \xi_{2}\right)$ in the plane $1-2$

$$
\begin{aligned}
& \chi(\boldsymbol{\xi}) \simeq f \varphi\left(\eta^{*}\right), \quad \varphi\left(\eta^{*}\right)=\frac{3\left(\sin \eta^{*}-\eta^{*} \cos \eta^{*}\right)}{\eta^{* 3}}, \\
& \eta^{*}=\sqrt{\left(\xi_{1} b_{1}\right)^{2}+\left(\xi_{2} b_{2}\right)^{2}}
\end{aligned}
$$


Regarding the matrix $\boldsymbol{K}^{0}$, it is diagonal $\boldsymbol{K}^{0}=\operatorname{diag}\left[k_{1} k_{2} k_{3}\right]$ in the same coordinate system. As a result, the components of tensor $\boldsymbol{H}$ read

$$
\begin{aligned}
& H_{i j}(\boldsymbol{\xi})=\delta_{i j}-\frac{2 k_{i} \xi_{i} \xi_{j}}{k_{1} \xi_{1}^{2}+k_{2} \xi_{2}^{2}+k_{3} \xi_{3}^{2}} \quad \forall \boldsymbol{\xi} \neq 0, \quad(i \text { not summed }) \\
& H_{i j}(\mathbf{0})=0
\end{aligned}
$$

To facilitate the study of aligned cracks parallel to the plane $1-2$, we rearrange the tensor $\boldsymbol{H}$ as follows

$$
\boldsymbol{H}(\boldsymbol{\xi})=\boldsymbol{L}-2 \boldsymbol{M}(\boldsymbol{\xi}), \quad \forall \boldsymbol{\xi} \neq 0
$$

with

$$
\begin{aligned}
& \boldsymbol{L}=\left[\begin{array}{ccc}
1 & 0 & 0 \\
0 & 1 & 0 \\
0 & 0 & -1
\end{array}\right], \\
& \boldsymbol{M}(\boldsymbol{\xi})=\frac{1}{k_{1} \xi_{1}^{2}+k_{2} \xi_{2}^{2}+k_{3} \xi_{3}^{2}}\left[\begin{array}{ccc}
k_{1} \xi_{1}^{2} & k_{1} \xi_{1} \xi_{2} & k_{1} \xi_{1} \xi_{3} \\
k_{2} \xi_{1} \xi_{2} & k_{2} \xi_{2}^{2} & k_{2} \xi_{2} \xi_{3} \\
k_{3} \xi_{1} \xi_{3} & k_{3} \xi_{2} \xi_{3} & -k_{1} \xi_{1}^{2}-k_{2} \xi_{2}^{2}
\end{array}\right]
\end{aligned}
$$

Using the properties of the characteristic function $\chi$ in Appendix, the tensor $\boldsymbol{C}$ given by (15) can be rewritten as

$$
\boldsymbol{C}=(1-f) \boldsymbol{L}-2 f^{-1} \sum_{\boldsymbol{\xi} \neq 0} \chi(-\boldsymbol{\xi}) \boldsymbol{M}(\boldsymbol{\xi}) \chi(\boldsymbol{\xi})=(1-f) \boldsymbol{L}-2 f \boldsymbol{S},
$$

with

$$
\boldsymbol{S}=\sum_{\boldsymbol{\xi} \neq 0} \boldsymbol{M}(\boldsymbol{\xi}) \varphi^{2}(\eta)
$$


In the crack limit, form factor $\chi($ or $\varphi)$ is independent of $\xi_{3}$ and we can define the tensor $\boldsymbol{G}\left(\boldsymbol{\xi}^{*}\right)$ as

$$
\boldsymbol{S} \rightarrow \sum_{\boldsymbol{\xi}^{*} \neq 0} \boldsymbol{G}\left(\boldsymbol{\xi}^{*}\right) \varphi^{2}\left(\eta^{*}\right), \quad \boldsymbol{G}\left(\boldsymbol{\xi}^{*}\right)=\sum_{\xi_{3}} \boldsymbol{M}(\boldsymbol{\xi})
$$

Using the identities in Appendix A, we have

$$
\begin{aligned}
G_{i j}\left(\boldsymbol{\xi}^{*}\right) & =\frac{a_{3}^{2} k_{i} \xi_{i} \xi_{j}}{4 k_{3}} \frac{\operatorname{coth}\left[\frac{a_{3}}{2} \sqrt{\left(k_{1} \xi_{1}^{2}+k_{2} \xi_{2}^{2}\right) / k_{3}}\right]}{\frac{a_{3}}{2} \sqrt{\left(k_{1} \xi_{1}^{2}+k_{2} \xi_{2}^{2}\right) / k_{3}}}, \quad i, j=1,2, \\
G_{33}\left(\boldsymbol{\xi}^{*}\right) & =-G_{11}\left(\boldsymbol{\xi}^{*}\right)-G_{22}\left(\boldsymbol{\xi}^{*}\right), \\
G_{13}\left(\boldsymbol{\xi}^{*}\right) & =G_{31}\left(\boldsymbol{\xi}^{*}\right)=G_{23}\left(\boldsymbol{\xi}^{*}\right)=G_{32}\left(\boldsymbol{\xi}^{*}\right)=0 .
\end{aligned}
$$

The diagonal matrix $\boldsymbol{C}$ given by (24) can be evaluated by

$$
\begin{aligned}
& C_{11} \simeq(1-f)-2 f s_{1} \quad C_{22} \simeq(1-f)-2 f s_{2}, \\
& C_{33} \simeq-(1-f)-2 f\left(s_{1}+s_{2}\right), \\
& C_{12}=C_{21}^{1}=C_{13}=C_{31}=C_{23}=C_{32}=0
\end{aligned}
$$

with $s_{1}$ and $s_{2}$ being the infinite sums

$$
s_{1}=\sum_{\boldsymbol{\xi}^{*} \neq 0} \varphi^{2}\left(\eta^{*}\right) G_{11}\left(\boldsymbol{\xi}^{*}\right), \quad s_{2}=\sum_{\boldsymbol{\xi}^{*} \neq 0} \varphi^{2}\left(\eta^{*}\right) G_{22}\left(\boldsymbol{\xi}^{*}\right) .
$$

Substituting into (18) and considering the limit when $f \rightarrow 0$ yield the result for $\boldsymbol{K}^{e}$. For example for superconductive cracks, or $\boldsymbol{A}=\boldsymbol{I}$, we have

$$
\boldsymbol{K}^{e}=\left[\begin{array}{ccc}
k_{1}\left(1+1 / s_{1}\right) & 0 & 0 \\
0 & k_{2}\left(1+1 / s_{2}\right) & 0 \\
0 & 0 & k_{3}
\end{array}\right]
$$


For insulating cracks, or $\boldsymbol{A}=-\boldsymbol{I}$, the effective conductivity becomes

$$
\boldsymbol{K}^{e}=\left[\begin{array}{ccc}
k_{1} & 0 & 0 \\
0 & k_{2} & 0 \\
0 & 0 & k_{3}\left(1-\left(s_{1}+s_{2}\right)^{-1}\right)
\end{array}\right]
$$

It can be noticed here that the presence of superconductive cracks does not change the conductivity in direction 3 normal to a crack, i.e the material is continuous across the crack but enhances the conductivity in the crack plane. On the contrary, insulating cracks reduce the conductivity in direction 3 but not in the crack plane. These results are physically consistent.

Another type of crack usually considered in geomechanics is the Poiseuille type crack where the fluid can flow through with a finite flow rate. We assume that the transport in that crack can be modeled using an equivalent constant conductivity $\boldsymbol{K}^{1}$ which scales with the inverse pore size, or $f \boldsymbol{K}^{1}=\boldsymbol{D}=$ cst. As shown by Pouya and $\mathrm{Vu}$ (2012), using constant $\boldsymbol{K}^{1}$ does not necessarily reproduce the same nonuniform local gradient field when the ellipsoidal heterogeneity collapses into a crack but rather the flow rate contribution of the whole crack. To the first order of approximation of $f$, we can write

$$
\boldsymbol{A}=\boldsymbol{I}-2 f \boldsymbol{K}^{0} \cdot \boldsymbol{D}^{-1}+o\left(f^{2}\right)
$$

For simplification, let us assume that $\boldsymbol{D}$ is aligned with $\boldsymbol{K}^{0}$, its principal components being $\tilde{k}_{1}, \tilde{k}_{2}, \tilde{k}_{3}$. Then the estimated conductivity reads

$$
\boldsymbol{K}^{e}=\left[\begin{array}{ccc}
k_{1}\left(1+\left(k_{1} / \tilde{k}_{1}+s_{1}\right)^{-1}\right) & 0 & 0 \\
0 & k_{2}\left(1+\left(k_{2} / \tilde{k}_{2}+s_{2}\right)^{-1}\right) & 0 \\
0 & 0 & k_{3}
\end{array}\right]
$$

We can see that when $\tilde{k}_{1} \rightarrow \infty$ and $\tilde{k}_{2} \rightarrow \infty$, the case of superconductive crack above is recovered. However, when $\tilde{k}_{1}$ is finite, the effective conductiv- 
ity is different and depends explicitly on the components of tensor $\boldsymbol{D}$.

In the special case where the matrix is isotropic in the plane $1-2, k_{1}=$ $k_{2}=k$ and the cracks are circular, the radii are related by $b_{1}=b_{2}=b$ and $a_{1}=a_{2}=a$. Then, the explicit expressions for $s_{1}$ and $s_{2}$ are

$$
s_{1}=s_{2}=s=\frac{\gamma}{2} \sum_{\eta^{*} \neq 0} \eta^{*} \operatorname{coth}\left(\gamma \eta^{*}\right) \varphi^{2}\left(\eta^{*}\right), \quad \gamma=\frac{a_{3}}{2 b} \sqrt{\frac{k}{k_{3}}}, \quad \eta^{*}=b \xi^{*}
$$

The quantity $s$ is the infinite lattice sum in $\boldsymbol{\xi}^{*}$ space (2D) with lattice spacing $2 \pi / a$ in both directions 1,2 . In practice, to compute numerically this sum, we truncate it to a certain range of $\xi^{*}$ (or equivalently $\eta^{*}$ ). On the other hand, the sums can be approximated analytically by keeping several leading terms plus a 1D continuous integral (To et al., 2013, 2016). This is because for high values of $\xi^{*}$ the terms in the sum fluctuate less and there are more lattice points around the given wavevector radius $\xi^{*}$. Explicitly, we have

$$
s \simeq \frac{\gamma}{4 f^{*}} \int_{\eta_{c}}^{\infty} \operatorname{coth}\left(\gamma \eta^{*}\right) \varphi^{2}\left(\eta^{*}\right) \eta^{* 2} d \eta^{*}+\frac{\gamma}{2} \sum_{0<\eta^{*}<\eta_{c}} \eta^{*} \operatorname{coth}\left(\gamma \eta^{*}\right) \varphi^{2}\left(\eta^{*}\right)
$$

where $\eta_{c}$ is the truncation value, used to determine the number of leading terms kept in the series, and $f^{*}=\pi b^{2} / a^{2}$ is the planar density of cracks. We also note that the function $\operatorname{coth}(x)$ is infinite at $x=0$ but converges exponentially to 1 , indeed, an asymptotic expansion of this function for large $x$ produces $\operatorname{coth}(x)=1+2 e^{-2 x}+2 e^{-4 x}+\mathcal{O}\left(e^{-6 x}\right)$. In addition, the convergence is fast, due to the exponential term. As a result, if $\eta_{c}$ is sufficiently large, we can make the approximation $\operatorname{coth}\left(\gamma \eta^{*}\right) \simeq 1$ and derive an analytical expression for the integral term in (35). Regarding the finite sum of (35), numerical tests show that for $\gamma>0.5$, it is sufficient to adopt two leading 
terms, i.e $\eta_{c}=4 \pi b / a$ to obtain satisfactory results. Posing $\epsilon=2 \pi b / a$, the explicit and simple analytical expression of $s$ is the following

$$
\begin{aligned}
& s \simeq 18 \gamma\left[\operatorname{coth}(\gamma \epsilon) \frac{(\sin \epsilon-\epsilon \cos \epsilon)^{2}}{\epsilon^{5}}+\operatorname{coth}(\gamma \sqrt{2} \epsilon) \frac{(\sin \sqrt{2} \epsilon-\sqrt{2} \epsilon \cos \sqrt{2} \epsilon)^{2}}{(\sqrt{2} \epsilon)^{5}}\right] \\
& +\frac{3 \gamma}{64 \epsilon^{3} f^{*}}\left[2 \sin ^{2} 2 \epsilon-4 \epsilon \sin 4 \epsilon+4 \epsilon^{2}(3-\cos 4 \epsilon)+8 \epsilon^{3}(\pi-2 \operatorname{Si}(4 \epsilon))\right] .
\end{aligned}
$$

Here we account for the analytical expression of $\varphi\left(\eta^{*}\right)$ and $\mathrm{Si}$ is the sine integral function.

\subsection{Random distribution of aligned penny shaped cracks}

Considering first $N$ identical ellipsoids randomly distributed in the volume $V$, the characteristic function $\chi$ becomes

$$
\chi(\boldsymbol{\xi})=\frac{f}{N} \varphi(\eta) \sum_{i=1}^{N} e^{-i \boldsymbol{\xi} \cdot c^{i}}
$$

where $\boldsymbol{c}^{i}$ are locations of the ellipsoid centers. In the infinite volume limit, the tensor $\boldsymbol{C}$ in (18) and the product $\chi(\boldsymbol{\xi}) \chi(-\boldsymbol{\xi})$ are equivalent to their ensemble averages, notation \langle\rangle$_{\text {ens }}$. Due to the relation to the structure factors defined as

$$
S(\boldsymbol{\xi})=\frac{1}{N}\left\langle\sum_{i=1}^{N} e^{-i \boldsymbol{\xi} \cdot c^{i}} \sum_{i=1}^{N} e^{i \boldsymbol{\xi} \cdot c^{i}}\right\rangle_{\text {ens }}
$$

the following connection can be established

$$
\boldsymbol{C}=(1-f) \boldsymbol{L}-\frac{2 f}{N} \sum_{\boldsymbol{\xi} \neq 0} \boldsymbol{M}(\boldsymbol{\xi}) \varphi^{2}(\eta) S(\boldsymbol{\xi})
$$

Next, we focus on the particular case where the ellipsoids collapse into cracks which are non overlapping circles $\left(b_{1}=b_{2}=b, b_{3}=0\right)$ with a distribution 
that is random and isotropic in plane $1-2$ and periodical along direction 3 with period $a_{3}$. The matrix is also isotropic in plane $1-2$, i.e. $k_{1}=k_{2}=k$, and the dimensions $a_{1}, a_{2}$ are very large. Consequently, the structure factor $S\left(\boldsymbol{\xi}^{*}\right)$ is associated to the planar wave vector $\boldsymbol{\xi}^{*}$ and depends only on its magnitude $S(\boldsymbol{\xi})=S\left(\boldsymbol{\xi}^{*}\right)=S\left(\xi^{*}\right)$. This will change the expression for $\boldsymbol{C}$ as follows:

$$
\boldsymbol{C}=(1-f) \boldsymbol{L}-\frac{2 f}{N} \sum_{\boldsymbol{\xi}^{*} \neq 0} \boldsymbol{G}\left(\boldsymbol{\xi}^{*}\right) \varphi^{2}\left(\eta^{*}\right) S\left(\xi^{*}\right)
$$

We note that, at the infinite volume limit $a_{1}, a_{2} \rightarrow \infty$, the $\boldsymbol{\xi}$ lattice with spacing $2 \pi / a_{1}$ and $2 \pi / a_{2}$ is infinitely dense. Consequently the lattice sum can be estimated by a continuous integral. In that case the quantities $s_{1}$ and $s_{2}$ in the previous section are:

$$
s_{1}=s_{2}=s=\frac{\gamma}{8 \pi^{2} \rho} \int \eta^{*} \operatorname{coth} \gamma \eta^{*} \varphi^{2}\left(\eta^{*}\right) S\left(\xi^{*}\right) d \boldsymbol{\xi}^{*}
$$

where $\rho=N /\left(a_{1} a_{2}\right)$ is the planar number density. Since the function in the $2 \mathrm{D}$ integral depends only on the radius $\xi^{*}$ ( or $\eta^{*}$ ), we can integrate on rings of radius $\xi^{*}$ and thickness $d \xi^{*}$ that $d \boldsymbol{\xi}^{*}=2 \pi \xi^{*} d \xi^{*}$ and convert it to $1 \mathrm{D}$ integral

$$
s=\frac{\gamma}{4 f^{*}} \int_{0}^{\infty} \operatorname{coth}\left(\gamma \eta^{*}\right) S\left(\xi^{*}\right) \varphi^{2}\left(\eta^{*}\right) \eta^{* 2} d \eta^{*}
$$

where $f^{*}=\pi \rho b^{2}$ is the planar crack density. We note that for a planar isotropic distribution, the structure factor $S\left(\xi^{*}\right)$ is related to the planar radial distribution function (rdf) $g(r)$ via the expression

$$
\begin{aligned}
& S\left(\xi^{*}\right)=1+\rho \int e^{-i \boldsymbol{\xi} \cdot \boldsymbol{r}}[g(r)-1] d \boldsymbol{r}= \\
& =1+2 \pi \rho \int_{0}^{\infty} J_{0}\left(r \xi^{*}\right)[g(r)-1] r d r
\end{aligned}
$$


where $J_{n}$ with $(n=0,1, \ldots)$ represents the Bessel function of the first kind and $n$th order. The above equations can be rewritten using the non dimensionless radius $\bar{r}=r / b$ and wavelength $\eta^{*}$ in the following form

$$
S\left(\xi^{*}\right)=1+2 f^{*} \mathcal{H}(g(b \bar{r})-1), \quad \mathcal{H}(\phi(\bar{r}))=\int_{0}^{\infty} J_{0}\left(\bar{r} \eta^{*}\right) \phi(\bar{r}) \bar{r} d \bar{r}
$$

with $\mathcal{H}(\phi(\bar{r}))$ the zero-order Hankel transform of the function $\phi(\bar{r})$ of variable $\bar{r}$ into a function of variable $\eta^{*}$.
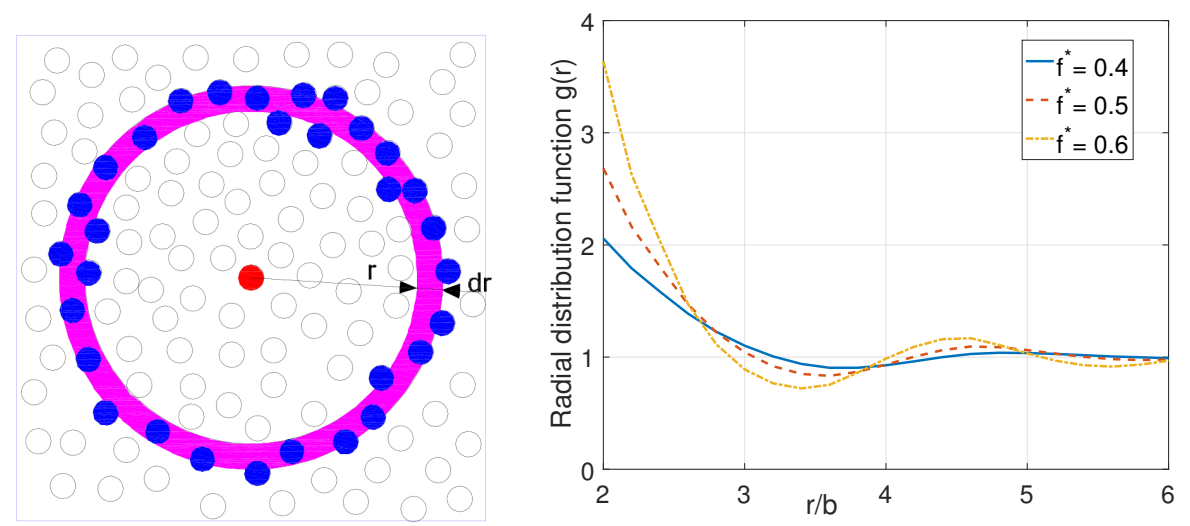

Figure 2: Random distribution of hard disks (left) and the radial distribution function $g(r)$ at different fraction $f^{*}$ obtained from MC simulations (Chae et al., 1969). The radial distribution function $g(r)$ is the probability of finding particle at distance $r$ from a reference particle.

Since our special system is constituted of identical nonoverlapping disks of diameter $2 b$, it is interesting and convenient to approach the problem from the statistics of disk centers in Fourier space as shown above. In this case, the effective properties depend on the distribution of circles via the parameters $g(r)$ or $S\left(\xi^{*}\right)$. These important quantities reflect the structure or the distribution of the circles. For example $g(r)$ shows how particles are distributed around a reference particle. It is known that $g(r)$ is sensitive to the algorithm of realizations of the samples (Rintoul and Torquato, 1997). Here, in 

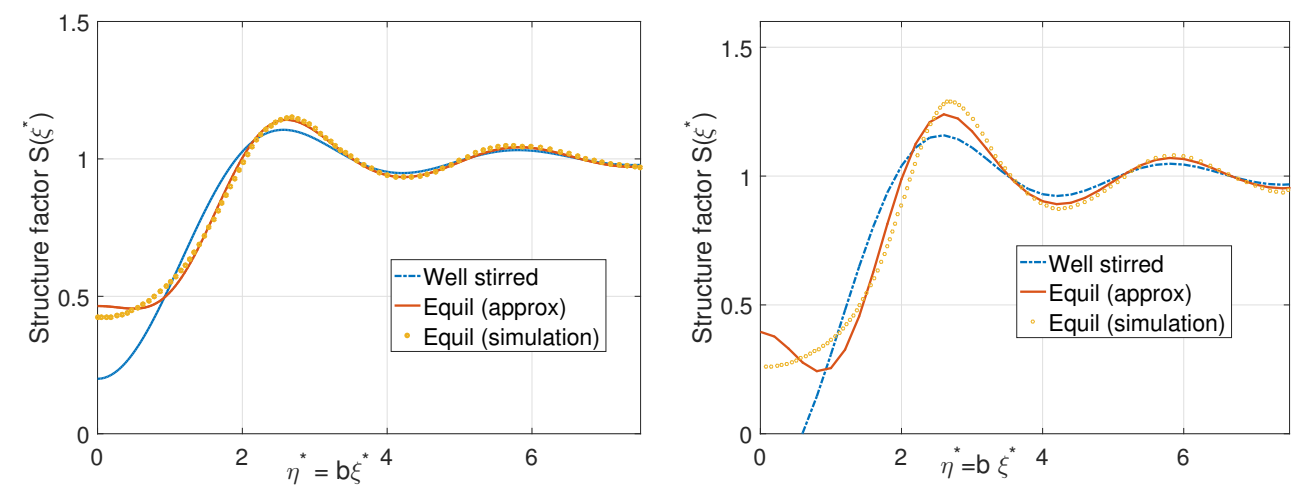

Figure 3: Structure factors $S\left(\xi^{*}\right)$ of equilibrium hard disk at $f^{*}=0.2$ (left) and $f^{*}=0.3$ (right). Comparisons between analytical approximations and particle simulations (de Macedo Biniossek et al., 2018).

this work, we examine equilibrium distributions which can be generated by Molecular Dynamics (MD) or Monte Carlo (MC) simulations and the structure functions $g(r)$ and $S(\xi)$ associated with this method. These methods are increasingly used nowadays as standard tools to generate random media. Different from the classical Random Sequential Addition (RSA) method (see e.g Kushch and Sangani, 2000), the MD and MC methods allow a particle random motion under interaction force without overlapping and thus help to construct samples with higher volume fractions.

Considering the well-stirred ( $w s)$ distribution relevant for small density $\left(f^{*} \leq\right.$ $0.1)$ system

$$
g(r)=g^{w s}(r)=\Theta(\bar{r}-2)
$$

with $\Theta$ being the Heaviside function, we can obtain the associated structure factor

$$
S\left(\xi^{*}\right)=S^{w s}\left(\xi^{*}\right)=1-4 f^{*} J_{1}\left(2 \eta^{*}\right) / \eta^{*}
$$


At moderate density, i.e. $f^{*}=0.2-0.3$, it can be approximated by (Buryachenko, 2007; Torquato, 2001)

$$
g(r)=g^{e q}(r) \simeq g^{w s}(r)+\frac{8 f^{*}}{\pi}\left[\cos ^{-1} \frac{\bar{r}}{4}-\frac{\bar{r}}{4} \sqrt{1-\frac{\bar{r}^{2}}{16}}\right] \Theta(4-\bar{r}) \Theta(\bar{r}-2)
$$

There is no analytical expression for the structure factor $S\left(\xi^{*}\right)$, but it is possible to calculate numerically $S\left(\xi^{*}\right)=S^{e q}\left(\xi^{*}\right)$ for given $\xi^{*}$ and compute the lattice sum $s$. As shown in Fig. 3, we can find that the approximation works very well for $f^{*}=0.2$. Some visible discrepancies appear at small frequencies in case $f^{*}=0.3$ due to the rough estimation of $g(r)$ at long range. However, the approximation reflects quite well the structure factor globally. It must be noticed also that the function $\operatorname{coth}\left(\gamma \eta^{*}\right) S\left(\xi^{*}\right) \varphi^{2}\left(\eta^{*}\right) \eta^{* 2}$ in the integral expression of $s$ vanishes at $\eta^{*}=0$. This reduces obviously the effect of the approximation at small frequencies in the evaluation of the structure factor on the evaluation of $s$.

At higher fraction $f^{*}$, we can use Baus-Colot (Baus and Colot, 1987) approximations or numerical results issued from $\mathrm{MD} / \mathrm{MC}$ simulation results (Chae et al., 1969; de Macedo Biniossek et al., 2018).

\section{Numerical applications}

\subsection{Periodic penny-shape cracks}

As a first example, we consider a penny shape crack of radius $b$ lying on the plane $x_{3}=0$ at the center of the unit cell of dimensions $a_{1}=1, a_{2}=1$ and $a_{3}=0.5$. The periodicity of the system in the plane $x_{1} x_{2}$ corresponds to the square lattice arrangement of the cracks. For comparison, a finite element computation has been performed using the mesh shown in Fig. 4. The conductivity of the anisotropic matrix is diag [ $\left.\begin{array}{llll}10 & 10 & 5\end{array}\right]$ in the same cartesian 


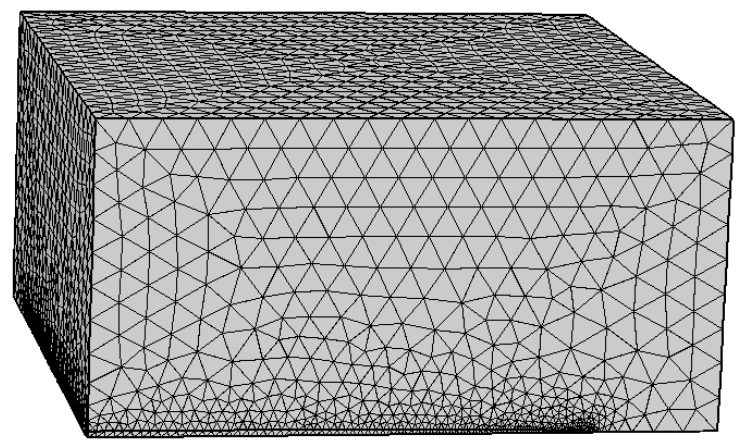

Figure 4: Finite element model of a single crack in a rectangular box. Due to the symmetry, only one eighth of the unit cell is presented. The crack is modelled as a flattened ellipsoid with different conductivity.

coordinate system. For the case of Poiseuille crack, its behavior is assumed to be isotropic with conductivity $\tilde{k}_{1}=\tilde{k}_{2}=\tilde{k}_{3}=1$. The radius $b$ of the crack is varied, resulting in the planar density $f^{*}=\pi b^{2} /\left(a_{1} a_{2}\right)$ varying from 0 to 0.6 . To compute the estimates in $(30,31,33)$, we use the analytical expressions (36) for the lattice sums. Numerical tests based on the analytical estimates are in excellent agreement with the Finite Element Method (FEM) simulations at moderate density $f^{*}$ below the percolation limit. For example, when $b=0.3$ and $b=0.4$ as present in Tab. 1 we find that the differences between the two methods are mostly 1-2\%. The largest recorded difference is $5 \%$ corresponding to the case of large insulating cracks when $b=0.4$. Noting that the maximal value that $b$ can take is 0.5 (percolation/coalescence limit), we can conclude about the accuracy of the estimation for the range of density.

Next we examine the variation of effective properties in terms of the quantities $f^{*}, \gamma$ and types of crack. As expected, results in Fig. 5 show that superconductive cracks increase the permeability in the direction parallel to the crack and insulating cracks decrease the permeability in the direction normal to the crack. The Poiseuille crack also increases slightly the perme- 


\begin{tabular}{|c|cc|cc|}
\hline & \multicolumn{2}{|c|}{$b=0.3$} & \multicolumn{2}{c|}{$b=0.4$} \\
Crack type & FEM & Est. & FEM & Est. \\
\hline Supercond. $\left(k_{1}^{e}\right)$ & 12.13 & 12.26 & 15.78 & 15.61 \\
Insulating $\left(k_{3}^{e}\right)$ & 4.49 & 4.46 & 3.80 & 3.60 \\
Poiseuille $\left(k_{1}^{e}\right)$ & 10.68 & 10.30 & 10.8 & 10.8 \\
\hline
\end{tabular}

Table 1: Comparisons of effective conductivity obtained from analytical estimates and FEM solutions for different crack types.

ability. The latter is less sensitive to the composite parameter $\gamma$. We note that small values of $\gamma$ correspond to a high crack density in $x_{3}$ direction, i.e the crack layers are close to each other, and high conductivity of the matrix in $x_{1}, x_{2}$ directions. As a result, the interaction between the cracks is strong. In the other extremes, when $\gamma$ is large, the influence of the cracks is weaker.

\subsection{Influence of planar crack distributions}

In this section, we study a set of penny shape cracks of radius $b$ distributed randomly and isotropically on the plane $x_{3}=0$. Our cell has thickness $a_{3}=0.5$ and is infinite along directions $x_{1}, x_{2}$. The conductivities of the matrix and Poiseuille crack are the same as for the single crack problem in Section 3.1.

In this case, the parameter $s$ which governs the effective properties of cracked materials depends on the fraction $f^{*}$ and $\gamma$. Here we have assumed that for each $f^{*}$, there is a unique structure factor $S\left(\xi^{*}\right)$ associated to the equilibrium system described in Section 2.4. To compute the integral $s$, we compare different approximations of $S\left(\xi^{*}\right)$ and MC simulation results for $g(r)$ tabulated in Chae et al. (1969) for $f^{*}=0.4-0.6$. The periodic case studied in Section 3.1 is also used for comparison.

From Table 2, we find that the discrepancies between different $s$ estimates are relatively small but follow the order: the WS results are higher than the 


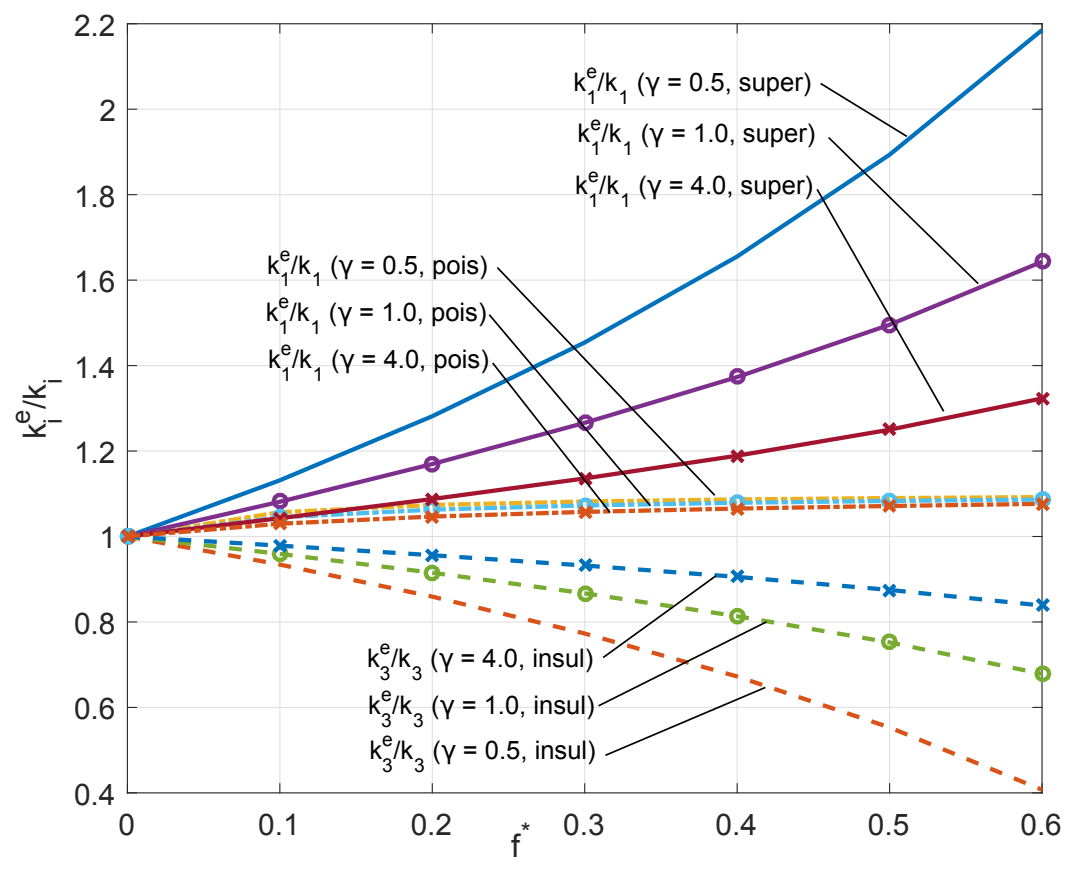

Figure 5: Dimensionless effective permeability $k_{i}^{e} / k_{i}$ for superconductive (super), insulating (insul) and Poiseuille (pois) cracks as functions of composite parameter $\gamma$ and planar density $f^{*}$. 


\begin{tabular}{|c|c|c|c|c|c|c|c|c|}
\hline \multirow[b]{2}{*}{$f^{*}$} & \multicolumn{3}{|c|}{$\gamma=0.5$} & \multirow[b]{2}{*}{ SQ } & \multicolumn{3}{|c|}{$\gamma=1.0$} & \multirow[b]{2}{*}{ SQ } \\
\hline & WS & Appr & $\mathrm{MC}$ & & WS & Appr & $\mathrm{MC}$ & \\
\hline 0.1 & 7.572 & 7.572 & - & 7.606 & 12.198 & 12.192 & - & 12.297 \\
\hline 0.2 & 3.534 & 3.532 & . & 3.553 & 5.828 & 5.817 & . & 5.898 \\
\hline 0.3 & 2.187 & 2.185 & . & 2.202 & 3.704 & 3.688 & - & 3.760 \\
\hline 0.4 & 1.514 & 1.511 & 1.509 & 1.526 & 2.642 & 2.621 & 2.608 & 2.681 \\
\hline 0.5 & 1.110 & 1.106 & 1.102 & 1.120 & 2.005 & 1.978 & 1.956 & 2.018 \\
\hline 0.6 & 0.841 & 0.836 & 0.829 & 0.844 & 1.581 & 1.548 & 1.513 & 1.555 \\
\hline$f^{*}$ & WS & $\begin{array}{c}\gamma=2.0 \\
\text { Appr }\end{array}$ & $\mathrm{MC}$ & SQ & WS & $\begin{array}{c}\gamma=4.0 \\
\text { Appr }\end{array}$ & $\mathrm{MC}$ & SQ \\
\hline 0.1 & 23.053 & 23.030 & 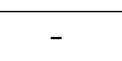 & 23.390 & 45.706 & 45.643 & - & 46.594 \\
\hline 0.2 & 11.162 & 11.114 & - & 11.405 & 22.211 & 22.086 & - & 22.782 \\
\hline 0.3 & 7.198 & 7.127 & - & 7.360 & 14.380 & 14.192 & - & 14.713 \\
\hline 0.4 & 5.216 & 5.122 & 5.087 & 5.289 & 10.464 & 10.214 & 10.147 & 10.577 \\
\hline 0.5 & 4.027 & 3.909 & 3.847 & 4.001 & 8.115 & 7.802 & 7.680 & 8.002 \\
\hline 0.6 & 3.234 & 3.093 & 2.997 & 3.094 & 6.548 & 6.173 & 5.988 & 6.188 \\
\hline
\end{tabular}

Table 2: Dependence of lattice sum $s$ on the planar crack density $f^{*}$, composite parameter $\gamma$ and hard disk distribution $g(r)$. Different RDFs of $g(r)$ are used: Well stirred (WS), approximation of equilibrium ensemble (Appr), Monte Carlo simulation (MC) and square lattice (SQ) arrangement in section 3.1. 
approximation results and the latter are higher than the $\mathrm{MC}$ results. The periodic distribution yields slightly higher results at small $f^{*}$ and smaller at high $f^{*}$. The difference between the highest and the smallest is of order $10 \%$ at the high density $f^{*}$. Since the relative changes $\left|\left(k_{i}^{e}-k_{i}\right) / k_{i}\right|$ in permeability according to $(30,31,33)$ are $1 /|s+1|$ for superconductive crack, $1 /|2 s-1|$

for insulating crack and $1 /\left|k_{1} / \tilde{k}_{1}+s+1\right|$, the maximal differences between the estimates is of order $6-7 \%$. It is also interesting to note that, although the periodic distribution is obviously oversimplifying the true random distribution, the overall properties predicted by the analytical estimates for the periodic case compare well with those obtained from the full statistical computation. This result is due to the approximation of an uniform polarization field in the crack, which is valid at small density. At higher density, the cracks interact strongly and higher order approximations could be used to improve the estimates (see e.g Nguyen et al., 2016; To et al., 2017).

\section{Concluding remarks}

In this work, estimates of effective permeability of porous materials containing ellipsoidal heterogeneities or penny shape cracks have been presented. The estimates are based on an approximation of the integral equation established for the polarization in the heterogeneities. The penny shape cracks are treated as special ellipsoids when the aspect ratio tends to 0 . The derivation of the transport properties of porous materials containing cracks in the context of FFT modelling is a new contribution to this field.

The main contribution of this work is the derivation of effective properties for both superconductive or insulating cracks from the distribution of cracks. We show that when the cracks are identical, lying in the same plane and aligned, statistics of particles based on the Fourier Transform can be used. Specifically, the overall permeability is connected to the planar structure factors of the particles. Examples including well-stirred and equilibrium 
distribution are also given. These results are of prime importance, because the planar structure factor is a quantity that can be built from an experimental observation of porous media.

Different from homogenization schemes like Mori-Tanaka (MT), Self Consistent (SC) schemes, etc.. based on Eshelby inclusions, the present estimate accounts for the interaction between the cracks due to the relative position of the cracks, via the periodicity condition and the radial distribution function. Although the results are derived for a system of aligned cracks lying on parallel planes, it can be extended to more general cases by several way. Estimates for multiple families of cracks can be obtained by applying classical MT, SC schemes to the above systems of cracks. On the other hand, more rigorous treatment accounting for the relative position of crack systems can be done following the same procedure presented in the paper. This method is more mathematically involved due to the presence of the cross correlation term with irreducible Lerch functions (see Appendix A), the complexity of the structure factor in $3 \mathrm{D}$ random cases and will be subject to future work.

\section{Acknowledgement}

This project is funded by the National Foundation for Science and Technology Development (NAFOSTED) under grant number 107.02-2016.05.

\section{Appendix A. Analytical expressions for infinite series}

For $n \in \mathbb{Z}$ and $c, m \in \mathbb{R}$, we have the following results for infinite sums

$$
\begin{aligned}
& \sum_{n=-\infty}^{\infty} \frac{e^{2 i \pi c n}}{n^{2}+m^{2}}=\frac{i}{m}\left[\Phi\left(e^{2 i \pi c}, 1, i m\right)-\Phi\left(e^{2 i \pi c}, 1,-i m\right)-\frac{1}{m}\right] \\
& \sum_{n=-\infty}^{\infty} \frac{e^{2 i \pi c n} n}{n^{2}+m^{2}}=\Phi\left(e^{2 i \pi c}, 1, i m\right)+\Phi\left(e^{2 i \pi c}, 1,-i m\right)
\end{aligned}
$$


where $\Phi$ is the Lerch transcendent function

$$
\Phi(z, s, a)=\sum_{n=0}^{\infty} \frac{z^{n}}{(n+a)^{s}}
$$

In the paricular case where $c=0$, we have

$$
\sum_{n=-\infty}^{\infty} \frac{1}{n^{2}+m^{2}}=\frac{\pi}{m} \operatorname{coth}(\pi m), \quad \sum_{n=-\infty}^{\infty} \frac{n}{n^{2}+m^{2}}=0
$$

From definition (4) of the wave vector $\boldsymbol{\xi}$, we can write

$$
\begin{aligned}
& \sum_{\xi_{3}=-\infty}^{\infty} \frac{1}{k_{1} \xi_{1}^{2}+k_{2} \xi_{2}^{2}+k_{3} \xi_{3}^{2}}=\sum_{\xi_{3}=-\infty}^{\infty} \frac{\frac{a_{3}^{2}}{4 \pi^{2} k_{3}}}{\frac{a_{3}^{2}\left(k_{1} \xi_{1}^{2}+k_{2} \xi_{2}^{2}\right)}{4 \pi^{2} k_{3}}+n_{3}^{2}} \\
& =\frac{a_{3}^{2}}{4 \pi^{2} k_{3}} \frac{\pi}{\sqrt{\frac{a_{3}^{2}\left(k_{1} \xi_{1}^{2}+k_{2} \xi_{2}^{2}\right)}{4 \pi^{2} k_{3}}}} \operatorname{coth} \pi \sqrt{\frac{a_{3}^{2}\left(k_{1} \xi_{1}^{2}+k_{2} \xi_{2}^{2}\right)}{4 \pi^{2} k_{3}}}
\end{aligned}
$$

and

$$
\sum_{\xi_{3}=-\infty}^{\infty} \frac{\xi_{3}}{k_{1} \xi_{1}^{2}+k_{2} \xi_{2}^{2}+k_{3} \xi_{3}^{2}}=0
$$

\section{Appendix B. Properties of characteristic functions in Fourier space}

The explicit expressions for operator $(\boldsymbol{H} * \chi)$ acting on the tensor $\boldsymbol{A}(\boldsymbol{x})$ are given as follows

$$
(\boldsymbol{H} * \chi) \boldsymbol{A}=\sum_{\boldsymbol{\xi}} e^{i \boldsymbol{\xi} \cdot \boldsymbol{x}} \boldsymbol{H}(\boldsymbol{\xi}) \sum_{\boldsymbol{\xi}^{\prime}} \chi\left(\boldsymbol{\xi}-\boldsymbol{\xi}^{\prime}\right) \boldsymbol{A}\left(\boldsymbol{\xi}^{\prime}\right),
$$

In the case where $\boldsymbol{A}$ is a constant, i.e $\boldsymbol{A}(\boldsymbol{\xi})=\mathbf{0} \forall \boldsymbol{\xi} \neq 0$ and $\boldsymbol{A}(\mathbf{0})=\boldsymbol{A}$ and $\chi$ is the characteristic function of a geometric region $\Omega$, the volume average 
over the volume $\Omega$ yields

$$
\langle(\boldsymbol{H} * \chi) \boldsymbol{A}\rangle_{\Omega}=f^{-1} \sum_{\boldsymbol{\xi}} \chi(-\boldsymbol{\xi}) \boldsymbol{H}(\boldsymbol{\xi}) \chi(\boldsymbol{\xi}) \boldsymbol{A}
$$

leading to the expression of $\boldsymbol{C}$ in (15).

Furthermore, due to the properties of the characteristic function $\chi=\chi^{2}$ and $\chi(0)=f$, we have the relations

$$
\left(\chi^{2}\right)(\boldsymbol{\xi})=\sum_{\boldsymbol{\xi}^{\prime}} \chi\left(\boldsymbol{\xi}-\boldsymbol{\xi}^{\prime}\right) \chi\left(\boldsymbol{\xi}^{\prime}\right)=\chi(\boldsymbol{\xi})
$$

and other associated properties

$$
\sum_{\boldsymbol{\xi}^{\prime} \neq 0} \chi\left(\boldsymbol{\xi}-\boldsymbol{\xi}^{\prime}\right) \chi\left(\boldsymbol{\xi}^{\prime}\right)=\chi(\boldsymbol{\xi})(1-f)
$$

Setting $\boldsymbol{\xi}=0$ in the above equation yields another property

$$
\sum_{\boldsymbol{\xi} \neq 0} \chi(-\boldsymbol{\xi}) \chi(\boldsymbol{\xi})=f(1-f)
$$

and leads to another expression of $\boldsymbol{C}$ in (24).

\section{References}

Barthélémy, J.F., 2009. Effective permeability of media with a dense network of long and micro fractures. Transp. Porous Med. 76, 153-178.

Baus, M., Colot, J.L., 1987. Thermodynamics and structure of a fluid of hard rods, disks, spheres, or hyperspheres from rescaled virial expansions. Phys. Rev. A 36, 3912.

Benveniste, Y., 1987. A new approach to the application of Mori-Tanaka's theory in composite materials. Mech. Materials , 147-157. 
Buryachenko, V., 2007. Micromechanics of heterogeneous materials. Springer Science \& Business Media.

Chae, D.G., Ree, F.H., Ree, T., 1969. Radial distribution functions and equation of state of the hard-disk fluid. J. Chem. Phys. 50, 1581-1589.

Dormieux, L., Kondo, D., 2004. Micromechanical approach to the coupling between permeability and damage. C. R. Mecanique 332, 135-140.

Eshelby, J., 1957. The determination of the elastic field of an ellipsoidal inclusion, and related problems. Proc. R. Soc. A 241, 376-396.

Fokker, P., 2001. General anisotropic effective medium theory for the effective permeability of heterogeneous reservoirs. Transp. Porous Med. 44, 205218.

Hansen, J.P., McDonald, I.R., 2006. Theory of simple liquids. Academic press.

Hashin, Z., 1988. The differential scheme and its application to cracked materials. J. Mech. Phy. Solids 36, 719-734.

Kröner, E., 1977. Bounds for effective moduli of disordered materials. J. Mech. Phys. Solids 25, 137-155.

Kushch, V., 1997. Microstresses and effective elastic moduli of a solid reinforced by periodically distributed spheroidal particles. Int. J. Solids Struct. 34, 1353-1366.

Kushch, V., Sangani, A.S., 2000. Conductivity of a composite containing uniformly oriented penny-shaped cracks or perfectly conducting discs. Proc. R. Soc. A 456, 683-699.

de Macedo Biniossek, N., Löwen, H., Voigtmann, T., Smallenburg, F., 2018. Static structure of active brownian hard disks. Journal of Physics: Condensed Matter 30, 074001. 
Michel, J., Moulinec, H., Suquet, P., 1999. Effective properties of composite materials with periodic microstructure: a computational approach. Comput. Method Appl. Mech. Eng. 172, 109-143.

Milton, G.W., 2002. The theory of composites. volume 6. Cambridge University Press.

Monchiet, V., Bonnet, G., 2013. A polarization-based fast numerical method for computing the effective conductivity of composites. Int. J. Numer. Methods H. 23, 1256-1271.

Mura, T., 1987. Micromechanics of defects in solids. Kluwer Academic Pub.

Nemat-Nasser, S., Hori, M., 2013. Micromechanics: overall properties of heterogeneous materials. volume 37. Elsevier Pub.

Nemat-Nasser, S., Yu, N., Hori, M., 1993. Solids with periodically distributed cracks. Int. J. Solids Struct. 30, 2071-2095.

Nguyen, H.L., To, Q.D., 2018. Conductivity of composites with multiple polygonal aggregates, theoretical estimates and numerical solutions from polarization series. Int. J. Eng. Sci. 123, 109-116.

Nguyen, M.T., Monchiet, V., Bonnet, G., To, Q.D., 2016. Conductivity estimates of spherical-particle suspensions based on triplet structure factors. Phys. Rev. E 93, 022105.

Ornstein, L., Zernike, F., 1914. Accidental deviations of density and opalescence at the critical point of a single substance. Proc. Acad. Sci. Amsterdam 17, 793-806.

Percus, J.K., Yevick, G.J., 1958. Analysis of classical statistical mechanics by means of collective coordinates. Phys. Rev. 110, 1.

Pouya, A., Vu, M.N., 2012. Fluid flow and effective permeability of an infinite matrix containing disc-shaped cracks. Adv. Water. Resour. 42, 37-46. 
Pozdniakov, S., Tsang, C.F., 2004. A self-consistent approach for calculating the effective hydraulic conductivity of a binary, heterogeneous medium. Water Resour. Res. 40.

Rintoul, M.D., Torquato, S., 1997. Reconstruction of the structure of dispersions. Journal of Colloid and Interface Science 186, 467-476.

Sævik, P.N., Berre, I., Jakobsen, M., Lien, M., 2013. A 3d computational study of effective medium methods applied to fractured media. Transp. Porous Med. 100, 115-142.

Shafiro, B., Kachanov, M., 2000. Anisotropic effective conductivity of materials with nonrandomly oriented inclusions of diverse ellipsoidal shapes. J. Appl. Phys. 87, 8561-8569.

Snow, D.T., 1969. Anisotropie permeability of fractured media. Water Resources Res. 5, 1273-1289.

To, Q.D., Bonnet, G., Hoang, D.H., 2016. Explicit effective elasticity tensors of two-phase periodic composites with spherical or ellipsoidal inclusions. Int. J. Solids Struct. 9495, $100-111$.

To, Q.D., Bonnet, G., To, V.T., 2013. Closed-form solutions for the effective conductivity of two-phase periodic composites with spherical inclusions. Proc. R. Soc. A 469, 1471-2946.

To, Q.D., Nguyen, M.T., Bonnet, G., Monchiet, V., To, V.T., 2017. Overall elastic properties of composites from optimal strong contrast expansion. Int. J. Solids Struct. 120, 245-256.

Torquato, S., 2001. Random heterogeneous materials: microstructure and macroscopic properties. Springer, Berlin.

Vu, M.N., Pouya, A., Seyedi, D.M., 2018. Effective permeability of threedimensional porous media containing anisotropic distributions of oriented 
elliptical disc-shaped fractures with uniform aperture. Adv. Water Resour. $118,1-11$.

Wertheim, M., 1963. Exact solution of the Percus-Yevick integral equation for hard spheres. Phys. Rev. Lett. 10, 321-323.

Zimmerman, R.W., 1996. Effective conductivity of a two-dimensional medium containing elliptical inhomogeneities. Proc. R. Soc. Lond. A 452, $1713-1727$. 